Trauma Berufskrankh 2017·19(Suppl 3):S289-S296 https://doi.org/10.1007/s10039-017-0314-2

Online publiziert: 18. Oktober 2017

๑) Springer Medizin Verlag GmbH 2017

CrossMark

S. T. Shafizadeh' - B. Bouillon' - J. H. Naendrup ${ }^{2}$ V. Jaecker' ${ }^{1}$ T. R. Pfeiffer ${ }^{1}$

${ }^{\prime}$ Klinik für Orthopädie, Unfallchirurgie und Sporttraumatologie, Lehrstuhl der Universität WittenHerdecke, Klinken der Stadt Köln - Klinikum Merheim Köln, Köln, Deutschland

${ }^{2}$ Universität zu Köln, Köln, Deutschland

\title{
Behandlung der akuten Kniegelenksluxation
}

letzungen der A. poplitea und des N. peroneus. Spontanrepositionen werden in ca. $50 \%$ der Fälle beobachtet, sodass eine kongruente femorotibiale Einstellung im Rahmen der Primärdiagnostik nicht selten vorgefunden wird und eine stattgehabte Luxationsverletzung nicht ausschließt [24].

Mit 0,2-0,3\% aller Luxationen stellt die Luxation des Kniegelenks eine seltene Verletzung dar [22, 26], von der hauptsächlich junge Männer im Alter von 18 bis 25 Jahren betroffen sind. Häufig sind Kniegelenksluxationen Folge von Hochrasanztraumen mit direktem Anprall gegen das Knie z. B. im Rahmen von „dashboard jnjuries“bei Verkehrsunfällen, Motorrad-/Fahrradstürzen oder Stürzen aus großer Höhe. Zunehmend häufig werden Knieluxationsverletzungen auch im Rahmen von Niedrigrasanztraumen beobachtet. Hierzu zählen v. a. Sportverletzungen. Während neurovaskuläre Begleitverletzungen bei Hochrasanztraumen häufig beobachtet werden, sind diese bei Niedrigrasanztraumen deutlich seltener [25].

Sogenannte Ultra-low-velocity-Luxationsverletzungen werden v. a. bei übergewichtigen Patienten beobachtet. Adipositas scheint das Risiko schwerwiegender Kniegelenksluxationen zu erhöhen. So reichen bereits Bagatelltraumen im täglichen Leben (Stolpern, Ausrutschen auf glattem Untergrund) aus schwerwiegende Verletzung des Kapselbandapparates zu verursachen. Wie bei Hochrasanztraumen sind adipöse Patienten mit einer Ultra-low-velocityLuxationsverletzung besonders gefährdet, neurovaskuläre oder knöcherne Begleitverletzungen zu erleiden [1].
Bilaterale oder offene Kniegelenksluxationen werden kaum beschrieben.

\section{Einteilung}

Die Einteilung der Kniegelenksluxation kann nach dem anatomischen Verletzungsmuster sowie nach Luxationsrichtung erfolgen. Basierend auf dem anatomischen Verletzungsmuster hat sich im klinischen Alltag die von Schenck konzipierte und nachfolgend überarbeitete Klassifikation etabliert ([23]; - Tab. 1).

Zum Verständnis des Verletzungsmusters ist ergänzend die Klassifikation nach der Luxationsrichtung hilfreich, die jedoch teilweise schwierig zu eruieren ist. Die von Kennedy entwickelte Einteilung unterteilt die Kniegelenksluxation nach Luxationsrichtung in 5 Typen: anterior, posterior, medial, lateral und "rotarory“ [9]. Dies hat sich jedoch nicht im klinischen Alltag durchgesetzt. Insgesamt kann die Einteilung der Knie-

\section{Tab. 1 Klassifikation nach Schenck}

\begin{tabular}{|c|c|}
\hline $\begin{array}{l}\text { Klassifi- } \\
\text { kation }\end{array}$ & Verletzungsmuster \\
\hline KD I & $\begin{array}{l}\text { HKB/VKB + posteromediale/ } \\
\text { posterolaterale Ecke }\end{array}$ \\
\hline KD ॥ & $H K B+V K B$ \\
\hline $\begin{array}{l}\mathrm{KD} \text { III } \\
(\mathrm{m} / \mathrm{l})\end{array}$ & $\begin{array}{l}\text { HKB + VKB + posteromediale } \\
(\mathrm{m}) / \text { posterolaterale (I) Ecke }\end{array}$ \\
\hline KD IV & $\begin{array}{l}\mathrm{HKB}+\mathrm{VKB}+\text { posteromediale }+ \\
\text { posterolaterale Ecke }\end{array}$ \\
\hline KD V & KD + intraartikuläre Fraktur \\
\hline \multicolumn{2}{|c|}{$\begin{array}{l}K D \text { „knee dislocation“, } m \text { medial, I late- } \\
\text { ral, } H K B \text { hinteres Kreuzband, VKB vorderes } \\
\text { Kreuzband } \\
\text { Durch Hinzufügen von C bzw. N wird das } \\
\text { Vorliegen einer Gefäß- bzw. Nervenverlet- } \\
\text { zung gekennzeichnet. }\end{array}$} \\
\hline
\end{tabular}



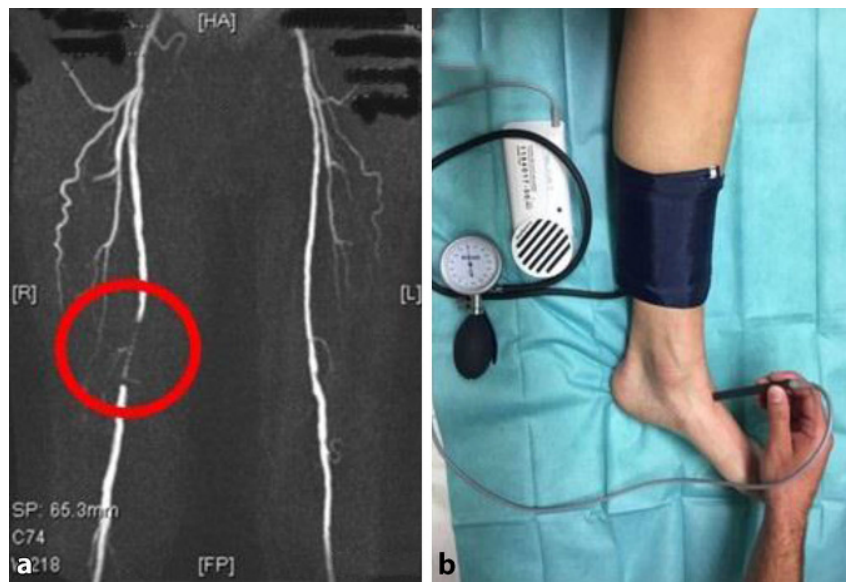

Abb. $1<$ a CT-Angiographie mit Verletzung der A. poplitea. b Doppleruntersuchung zur Bestimmung des krurobrachialen Index (CBI)/ Ankle-Brachial-Index (ABI)

gelenksluxationen aufgrund der großen Bandbreite an verletzten Strukturen und Begleitverletzung nur als annähernde Beschreibung der Verletzung genutzt werden.

\section{Begleitverletzung}

Aufgrund der großen Krafteinwirkung, die nötig ist, um eine Kniegelenksluxation zu verursachen, sowie der anatomisch engen Lagebeziehung von Gefäßen und Nerven, sind neurovaskuläre Begleitverletzungen häufig und haben maßgeblichen Einfluss auf die Prognose [3].

\section{Gefäßbeteiligung}

Der manifeste Verschluss der A. poplitea im Rahmen der Kniegelenksluxation oder auch okkulte Verletzungen im Sinne eines Intimaschadens mit protrahiertem Verschluss macht die Verletzung zu einer potenziell extremitätgefährdenden Verletzung.

Wie häufig Gefäßverletzungen infolge von Luxationstraumen des Kniegelenks auftreten, ist letztlich nicht ganz klar. Bei teilweise nur geringen Studienkollektiven werden vaskuläre Begleitverletzungen bei 3-30\% angegeben [18]. In einer Auswertung von 8050 Kniegelenksluxationen wurde bei 3,3\% der Patienten eine Gefäßbeteiligung beobachtet, wobei lediglich $13 \%$ dieser Gefäßverletzungen operativ behandelt werden mussten [18]. Da eine Ischämiezeit $>8 \mathrm{~h}$ das Risiko für eine Amputation signifikant erhöht und mit einer signifikanten Verschlechterung des Outcomes einhergeht, ist schon bei Verdacht auf Vorlie- gen einer Knieglenkluxationsverletzung sicherzustellen, dass keine Gefäßbeteiligung vorliegt [20].

Diagnostisch sind zunächst die klinische Untersuchung sowie die Erhebung des krurobrachialen Index (CBI)/AnkleBrachial-Index (ABI) (• Abb. 1) zu veranlassen. Auch wenn mehrere Studien zeigen konnten, dass interventionsbedürftige Intimaläsionen und Dissektionen bei normalem Pulsstatus und CBI $>0,9$ ausgeschlossen werden können, ist darauf hinzuweisen, dass die Erhebung des CBI und ABI nicht selten schmerzbedingt nur eingeschränkt möglich ist. Demzufolge ist die Indikation zur erweiterten Gefäßdiagnostik mittels CT-Angiographie bzw. konventioneller Angiographie immer dann großzügig zu stellen, wenn klinisch der Verdacht auf einen Gefäßverschluss bzw. Intimaschaden besteht, der CBI $<0,9$ ist oder wenn der CBI klinisch nicht sicher zu bestimmen ist (• Abb. 1). Die CTA (CTAngiographie) hat gegenüber der klassischen Angiographie den Vorteil der geringeren Invasivität und Strahlenbelastung.

$\mathrm{Da}$ Kniegelenksluxationsverletzungen selten sind, eignen sich Standardarbeitsanweisungen (SOPs; - Abb. 2), um Handlungsabläufe zu optimieren und einen gefäßchirurgischen Eingriff bei Nachweis einer durchblutungsstörenden Gefäßverletzung nicht zu verzögern.

Liegt eine interventionsbedürftige Gefäßverletzung vor, ist eine sofortige Reperfusion zu gewährleisten. Neben der Reperfusion ist eine Gelenkstabilisierung zu gewährleisten, um sekundäre instabilitätsbedingte Perfusionsstörungen zu vermeiden. Das Ausmaß der gelenkstabilisierenden Maßnahmen hängt daher wesentlich von der Ischämiezeit und den zu erwartenden Reperfusionsschäden ab, und es gelten grundsätzlich die Prinzipien des „damage controls“.

Neben einem primären ischämiebedingten Kompartmentsyndrom ist insbesondere auch auf ein sekundäres reperfusionsbedingtes Kompartmentsyndrom hinzuweisen. So sollte im Zweifel immer eine Kompartmentspaltung erfolgen.

\section{Nervenbeteiligung}

Die Prävalenz von Verletzungen des N. peroneus communis im Rahmen von Kniegelenksluxationen wird mit $16-40 \%$ angegeben, wobei diese am häufigsten mit Verletzungen der posterolateralen Ecke einhergehen [16]. Der $\mathrm{N}$. peroneus verläuft entlang des Biceps femoris und zieht zum posterioren Anteil des Caput fibulae. Diese anatomische Lage bedingt seine Verletzungsprädisposition, insbesondere bei Varus- und Hyperextensionstraumen. Im Gegensatz dazu stellen Verletzungen des N. tibialis eine Rarität dar. Die Verletzungsschwere kann von Nervendehnung (Neurapraxie) bis zur vollständigen Zerreißung reichen. Der sich in einen superfizialen und tiefen Ast aufteilende N. peroneus innerviert die Fußheber (M. peroneus longus und brevis, M. tibialis anterior, M. extensor hallucis longus, M. extensor digitorum longus). Bei Verdacht auf eine Kniegelenksluxation sollten daher immer die aktive Dorsiflexion des Fußes, der Großzehen sowie das sensible Innervationsgebiet des N. peroneus am Fußrücken zwischen 1. und 2 Strahl getestet werden.

Es empfiehlt sich diese mittels Kraftgrade nach Janda (0-5) zu dokumentieren. Die Magnetresonanztomographie (MRT) kann genutzt werden, um Verletzungen des N. peroneus zu bestätigen, ein perineurales Hämatom oder eine Zerreißung zu identifizieren sowie das Ausmaß der Verletzung der posterolateralen Ecke (PLC) zu bestimmen. Elektrophysiologische Untersuchungen (Elektromyographie [EMG] und Nervenleitgeschwindigkeit [NLG]) sollten bei funktionellen Einschränkungen der Fußhebung erstmals 
nach 6 Wochen zur Bestimmung der Baseline erfolgen. Ebenso sollten nach 3, 6 und 12 Monaten Verlaufsuntersuchungen zur Bestimmung des Regenerationspotenzials erfolgen.

Mit einer Spitzfußprophylaxe mittels Schiene und Physiotherapie sollte sofort nach Diagnosestellung begonnen werden. In der Literatur ist eine spontane Zurückbildung der Peroneusläsion in 14-56 \% der Fälle beschrieben, wobei v. a. junges Alter (<30 Jahre) zum Zeitpunkt der Verletzung mit höheren Raten spontaner Rückbildung verbunden ist.

Bei klinischem Verdacht der Verletzung des N. peroneus sind eine frühzeitige operative Darstellung und Dekompression empfehlenswert. Hierbei sind die vollständige Darstellung des Verlaufes, Entlastung des Hämatoms sowie eine Neurolyse indiziert. Bei Revision des $\mathrm{N}$. peroneus ist anatomische Rekonstruktion insbesondere der posterolateralen Ecke ggf. in Kombination mit einem Ligament-Bracing oder einer Bandersatzplastik zu empfehlen. Die Gefahr von iatrogenen Verletzungen des N. peroneus bei Folgeeingriff nach Neurolyse ist durch einen veränderten Verlauf und Narbenbildung deutlich erhöht. Daher sollte eine definitive Versorgung bereits im ersten Eingriff angestrebt werden. Bei kurzen Defektstrecken des N. peroneus sollte eine primäre Naht angestrebt werden. Ist dies nicht möglich, sollten die Nervenenden mit einem nichtresorbierbaren Faden markiert werden, sodass ggf. im Verlauf eine Nerventransplantation vorgenommen werden kann. Bei vollständiger Zerreißung des N. peroneus mit größerer Defektstrecke ( $a b$ ca. $>7 \mathrm{~cm}$ ) kann die Verbesserung der Fußhebung und des Gangbildes sowie der Patientenzufriedenheit z. B. durch eine Sehnentransposition der Tibialisposterior-Sehne ggf. in Kombination mit einer Transposition der Peroneuslongus-Sehne erreicht werden [15]. Aus unserer Erfahrung sollte dieser Eingriff erst nach neurologischem Ausschluss des Regenerationspotenzials, frühestens 1 Jahr nach Trauma, erfolgen.

Trauma Berufskrankh 2017 • 19 (Suppl 3):S289-S296

https://doi.org/10.1007/s10039-017-0314-2

(c) Springer Medizin Verlag GmbH 2017

\section{S. T. Shafizadeh · B. Bouillon · J. H. Naendrup - V. Jaecker · T. R. Pfeiffer \\ Behandlung der akuten Kniegelenksluxation}

Zusammenfassung

Kniegelenksluxationen stellen seltene, aber schwerwiegende Kniegelenksverletzungen dar. Da die Verletzung oftmals im Rahmen von Hochrasanztraumen entsteht, sind häufig junge aktive Patienten betroffen. Dabei sind kombinierte Verletzungen des vorderen und hinteren Kreuzbandes mit Beteiligung des medialen bzw. lateralen Kapselbandapparates typische Verletzungsmuster. Das frühzeitige Erkennen und die Behandlung von neurovaskulären Begleitverletzungen sind therapieentscheidend und haben maßgeblichen Einfluss auf das Outcome. Röntgen, CT-Angiographie, Magnetresonanztomographie und Narkoseuntersuchung unter Durchleuchtung sind therapieweisende diagnostische Untersuchungen. Nach aktueller Datenlage hat das operative Vorgehen Vorteile gegenüber dem konservativen Vorgehen. Maßgeblich für die Wahl des Operationsverfahrens ist das Ausmaß der Gesamtverletzungsschwere. Ziel der operativen Versorgung ist in erster Linie die anatomisch korrekte femorotibiale Einstellung und
Stabilisierung des Kniegelenks. Im Rahmen von Polytraumen oder bei Vorliegen von Gefäßverletzungen ist die Anlage eines Fixateur externe zu empfehlen. Ansonsten haben rekonstruktive Operationsverfahren in den ersten 14 Tagen nach Verletzung Vorteile gegenüber verzögerten Rekonstruktionsverfahren. Rekonstruktionsstrategien und -techniken müssen jeweils individuell an das Verletzungsmuster angepasst werden und hängen unter anderem auch von klinikabhängigen Ressourcen und der Erfahrung des Chirurgen ab. Trotz optimaler Versorgung und Nachbehandlung verbleiben nicht selten Restinstabilitäten, Funktionssowie Belastungseinschränkungen. Das Risiko für die Entwicklung einer Kniegelenksarthrose ist zudem deutlich erhöht.

\section{Schlüsselwörter}

Bandrekonstruktion · Komplexverletzung · Multiligamentverletzung · Hochrasanztraumen. Operation

\section{Treatment of acute knee dislocations}

\section{Abstract}

Dislocation of the knee is a rare but severe injury. Knee dislocations occur mainly in young active male patients mainly due to high velocity injury mechanisms. Combined injuries of the anterior and posterior cruciate ligaments associated with injuries of the medial or lateral collateral ligaments are typical injury patterns in these cases. The early recognition and treatment of accompanying neurovascular injuries must be carefully considered and are decisive for the prognosis. Computed tomography angiography (CTA), $\mathrm{X}$-radiography, magnetic resonance imaging (MRI) and fluoroscopic examination with the patient under anesthesia indicate the injury pattern and guide the treatment algorithm. According to current data conservative treatment seems to result in less favorable outcomes compared to surgical treatment. The decision-making process is influenced by multiple factors including injury pattern and associated injuries. The main aim of operative approaches is anatomically correct femorotibial alignment and stabilization of the knee joint. In the case of polytrauma or the presence of vascuar injuries, external fixation is recommended. Furthermore, reconstructive operative procedures in the first 14 days have advantages over delayed reconstruction procedures. Reconstruction strategies and techniques must be individually adapted to the injury pattern and are dependent among other things on hospital resources and experience of the surgeon. Despite optimal treatment and follow-up it is not uncommon for residual instability, functional and loading impairments to occur. Increased rates of early onset of arthrosis are to be expected.

\section{Keywords}

Ligament reconstruction - Complex injuries . Multiligament injuries - High velocity trauma . Surgery 


\section{Übersicht}

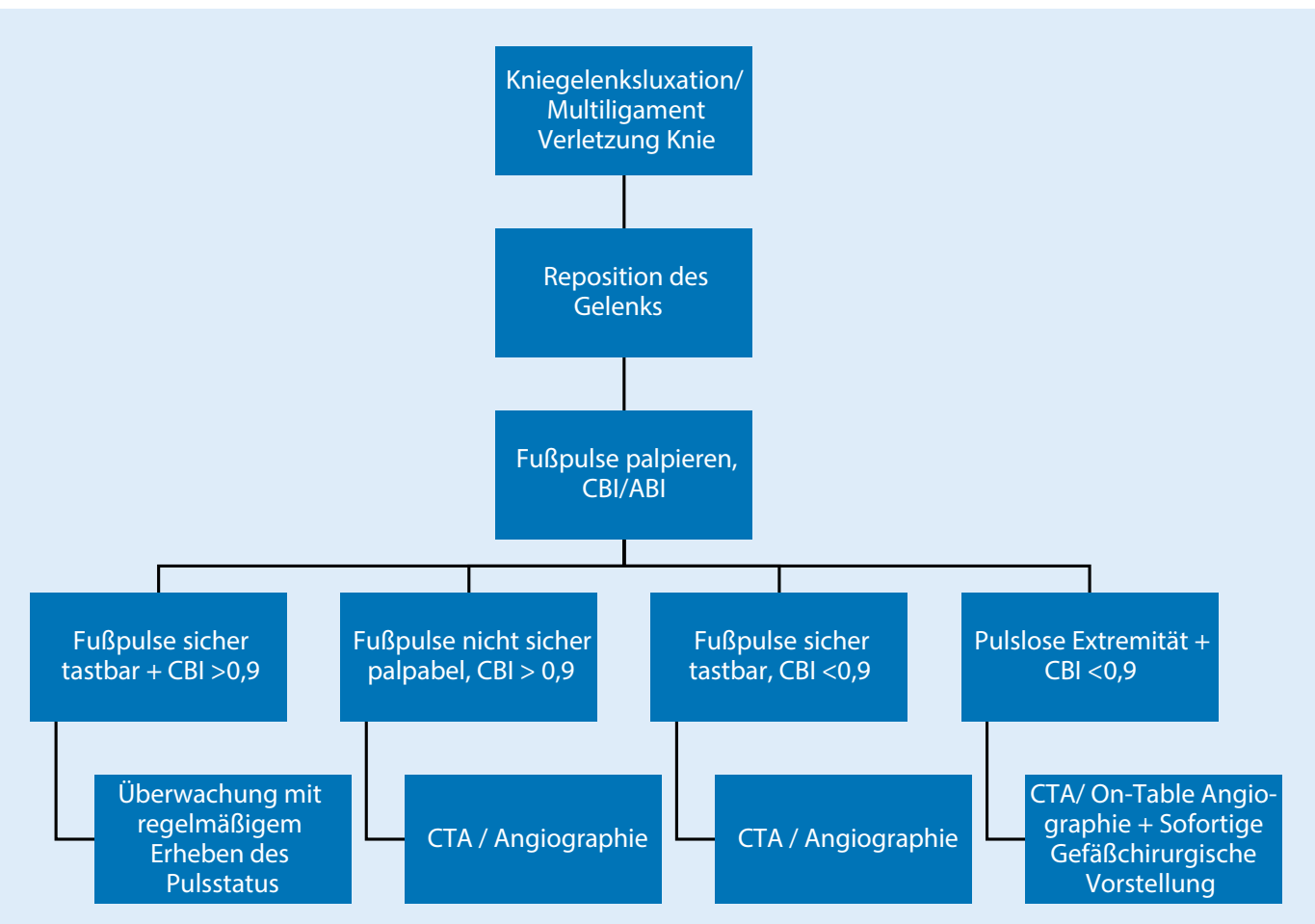

Abb. $2<$ Flowchart zur Diagnostik von Gefäßbeteiligung bei Kniegelenksluxationen. $C B I / A B I$ krurobrachialer Index/ Ankle-Brachial-Index, CTA CT-Angiographie
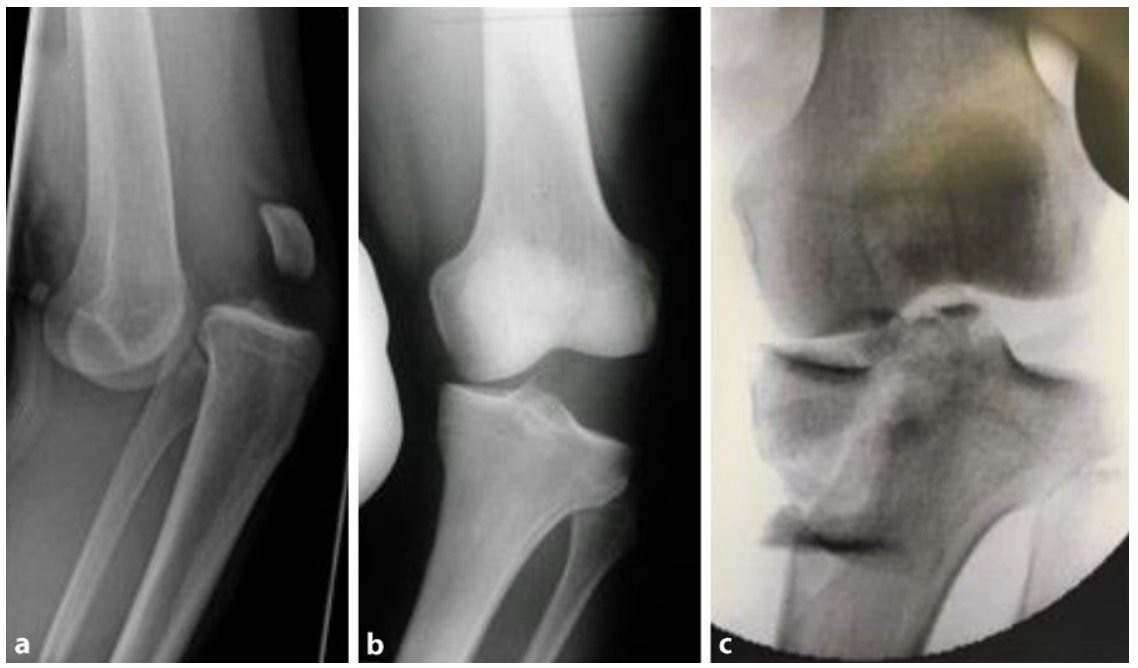

Abb. 3 A Röntgenbilder. a Anteriore Kniegelenksluxation im seitlichen Röntgenbild. b Grad-3-Varusinstabilität nach Kniegelenksluxation. c Mediale Tibiakopffraktur nach Kniegelenksluxation

\section{Bildgebung}

Diverse bildgebende Verfahren kommen in der Diagnostik der Knieluxation zur Anwendung. Die klassische Röntgenaufnahme hat dabei einen nicht zu unterschätzenden Stellenwert. Das frühzeitige Erkennen einer Fraktur und Fehlstellungen stehen hierbei im Vordergrund (• Abb. 3).

Die MRT bietet eine hohe Sensitivität insbesondere in der Diagnostik von
Weichteilverletzungen der Kreuzbänder, Menisken, der medialen wie lateralen Gelenkkapsel und des Knorpels. Dies verbessert die Möglichkeit der präoperativen Planung, jedoch besteht nur geringe Korrelation zwischen klinischer Instabilität und MR-Bildgebung [19]. Auch kann häufiger eine Unterschätzung der Instabilität nach Beurteilung der MR-Bildgebung beobachtet werden.

Gehaltene Aufnahmen in Narkose können hingegen die sagittale (a.-p.) und koronare (Varus-Valgus) Instabilität objektivieren und quantifizieren (- Abb. 4). Hierzu führen wir in unserer Klinik präoperativ unter Narkose sowohl gehaltene Aufnahmen im a.-p.Strahlengang der verletzten und gesunden Seite in $0^{\circ}$ und $20^{\circ}$ ohne Stress, in $0^{\circ}$ und $20^{\circ}$ unter Varusstress sowie in $0^{\circ}$ und $20^{\circ}$ unter Valgusstress sowie streng seitliche Aufnahmen neutral, in vorderer und hinterer Schublade durch. Erst nach Durchführung der dynamischen fluoroskopischen Stabilitätsuntersuchung kann eine abschließende Klassifikation der Luxationsverletzung erfolgen.

Bei intraartikulären Frakturen ist die CT sowohl zur präoperativen Planung der Osteosynthese als auch zur postoperativen Stellungskontrolle ein wichtiges Hilfsmittel.

\section{Therapie der Knieluxation}

Aufgrund der Heterogenität und Varianz der Verletzungsschwere von multiligamentären Kniegelenkverletzungen sind die möglichen Therapieoptionen groß, und dementsprechend ist die Evidenz aufgrund geringer Patientenzahlen limitiert. Ziel in der Behandlung von Kniegelenksluxationen, muss das Wiederer- 

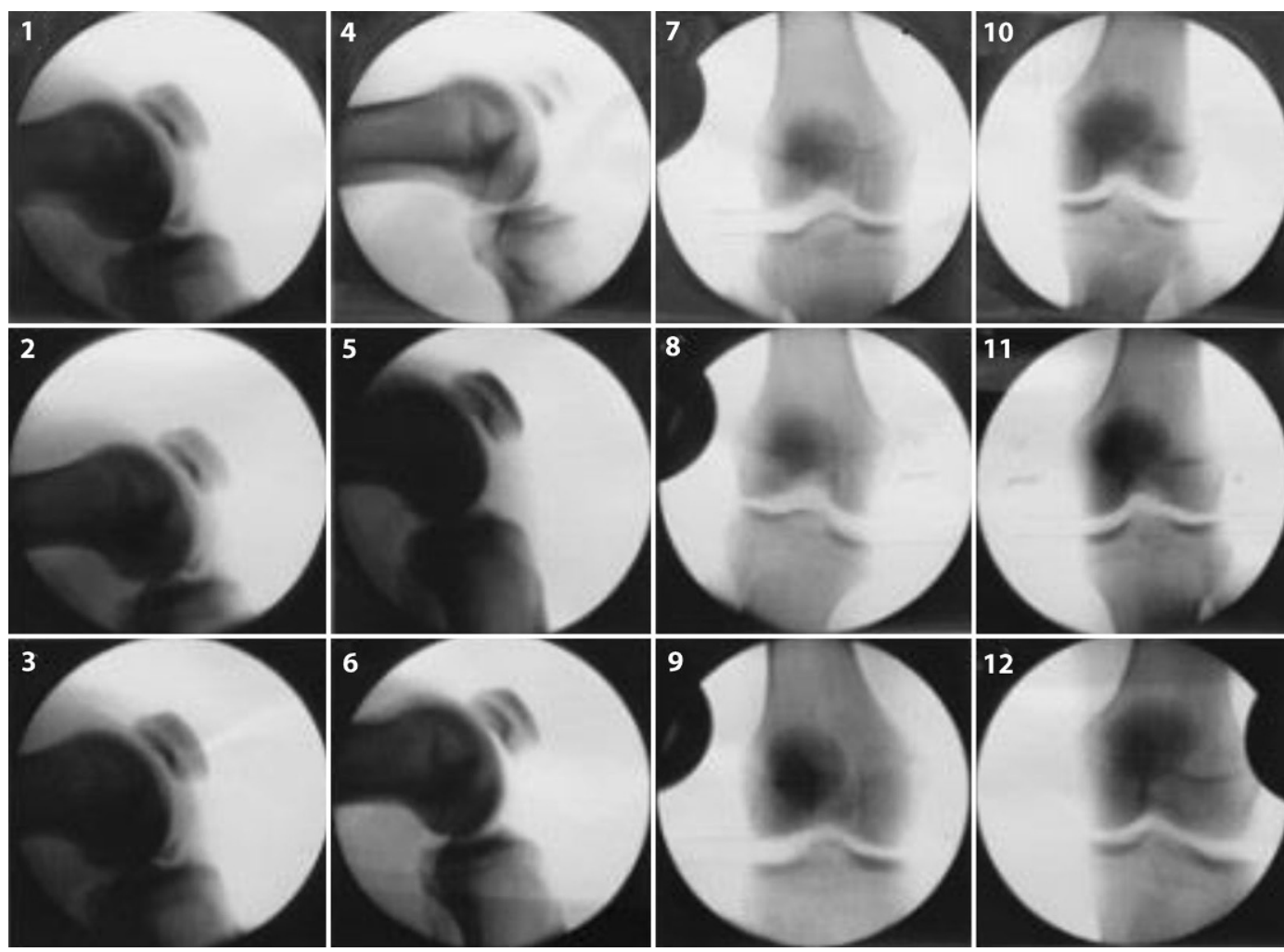

Abb. $4<$ Praeoperative Narkoseuntersuchung unter Durchleuchtung zur Bestimmung des Ausmaßes der zentralen (1-3) und periphären Instabilität (7-9) des betroffenen Kniegelenkes, sowie Darstellung der individuellen Laxizität der gesunden Seiten in der sagittalen (4-6) und koronaren Ebene (10-12)

langen eines stabilen, belastbaren funktionsfähigen Kniegelenks sein.

\section{Operatives versus konservatives Vorgehen}

Mehrere systematische Reviews und Metaanalysen konnten deutlich den Vorteil desoperativen Vorgehens im Vergleich zu konservativen Therapieschemata zeigen. So sind Patientenzufriedenheit, Funktionsscore, verbleibende Instabilität, Kontrakturen, erfolgreiche Wiedereingliederung in den Beruf und Rückkehr zum Sport signifikant besser nach operativem Vorgehen [11, 21]. Das nichtoperative Vorgehen kann bei schwer polytraumatisierten Patienten, Patienten mit schweren Komorbiditäten und/oder bei nur geringen Instabilitätsgraden in Erwägung gezogen werden. So können KD („knee dislocation“)-I-Verletzungen mit Beteiligung des medialen Kapselbandapparates teilweise konservativ mittels Orthese behandelt werden. Bei höhergradigen Instabilitäten (KD II) wird in unserem Traumazentrum ein frühzeitiges operatives Vorgehen empfohlen.

\section{Operationszeitpunkt}

Das operative Ergebnis der gelenkstabilisierenden Rekonstruktion hängt unter anderem vom Operationszeitpunkt ab.

Hierbei konkurriert eine sofortige (akute) definitive Versorgung aller verletzten Strukturen mit einer Versorgung im Intervall und mit einem zweizeitigen Vorgehen (verzögertes Vorgehen). Wenngleich keine evidenzbasierten Empfehlungen zum optimalen Zeitpunkt der Rekonstruktion existieren und dieser noch intensiv diskutiert wird, ist eine rekonstruktive Stabilisierung aus eigener Erfahrung so früh wie möglich, innerhalb der ersten 2 Wochen nach Verletzung, zu empfehlen. Während anatomische Rekonstruktionen in den ersten 2 Wochen meist gut möglich sind, ist die anatomische Präparation der verletzten Strukturen und ihre Rekonstruktion schon ab der dritten Woche nach Verletzung narbenbedingt deutlich erschwert. Als akute Versorgung wird daher meist eine Versorgung innerhalb von 3 Wochen empfohlen.

Eine absolute sofortige Operationsindikation stellen geschlossen nicht repositionierbare Luxationen, Luxations- verletzungen mit durchblutungskompromittierender Gefäßbeteiligung sowie offene Verletzungen dar.

Ebenfalls sollte bei Frakturen und Avulsionsverletzungen eine frühzeitige Versorgung angestrebt werden [12].

Die Studienergebnisse bezüglich sofortigem definitivem und zweizeitigem Vorgehen sind zum Teil widersprüchlich. Zum einen werden höhere Raten an Gelenksteife und verbleibender Instabilität beschrieben [17], zum anderen werden ein besseres subjektives Outcome und bessere Stabilität nach akuter Versorgung im Vergleich zum zweizeitigen Vorgehen berichtet [5, 7].

\section{Operationsstrategie}

Sofern die Entscheidung eines rekonstruktiven Verfahrens getroffen wurde, sollte zunächst das Ausmaß der zentralen und peripheren Instabilitäten im Rahmen der Narkoseuntersuchung im Seitenvergleich erhoben werden.

Der Umfang der Rekonstruktion und die Operationsstrategie erfordern die Berücksichtigung vieler Faktoren, und nicht immer ist eine vollständige, einzeitige Rekonstruktion möglich bzw. 


\section{Infobox 1 Behandlungsempfeh- lung des DGU (Deutsche Gesell- schaft für Unfallchirurgie)/ÖGU (Ös- terreichische Gesellschaft für Un- fallchirurgie)-Leitlinien-Komitees (AWFM [Arbeitsgemeinschaft der Wissenschaftlichen Medizinischen Fachgesellschaften]-Leitlinie)}

- Auch bei normalem Pulsstatus haben ca. $9 \%$ der Patienten nach Knieluxation angiographisch nachweisbare arterielle Intimaläsionen oder Stenosen! Daher ist die Gefäßdiagnostik nach Knieluxation obligat.

- Die konservative Therapie nach Kniegelenksluxation vom Typ 2-5 nach Schenk führt meist zu schlechten klinischen Ergebnissen.

- Bei kapseligen Läsionen kann der intrakompartimentelle Druck im Unterschenkel durch die Arthroskopie erhöht werden. Bei schweren Weichteilschäden oder drohendem Kompartmentsyndrom ist die Arthroskopie in der Akutphase kontraindiziert.

- Falls nach Knieluxation eine Früharthroskopie durchgeführt wird, sollte dies nur bei unkritischen Weichteilverhältnissen und mit wenig Wasserdruck erfolgen.

- Aufgrund der ausgeprägten Instabilität dieses Luxationstyps ist die Indikation zur Anlage eines Fixateur externe großzügig zu stellen. Die Stellung des Kniegelenks im Fixateur externe sollte radiologisch kontrolliert werden.

- Bei Verletzungen des hinteren Kreuzbandes auch im Rahmen von Multiligamentverletzungen sollte die Physiotherapie innerhalb der ersten 6 Wochen sehr zurückhaltend durchgeführt werden. Empfehlenswert sind das Tragen einer Hinteres Kreuzband(HKB)-Orthese und die Beübung in Bauchlage aus der Orthese mit eingeschränktem Bewegungsumfang.

- Auch bei aktuell bestmöglicher operativer Versorgung kann eine chronische Instabilität des Kniegelenks resultieren.

(Aus der AWMF-Leitlinie S1, letztes Update 10.06.2014)

sinnvoll. Grundsätzlich sind Gefäßverletzungen und Frakturen vorrangig zu adressieren. Liegen lediglich ligamentäre bzw. Kapselbandverletzungen vor, haben die peripheren Vorrang vor der Stabilisierung der zentralen Verletzungen, die ggf. zweizeitig adressiert werden können.

\section{Arthroskopie}

Der Stellenwert der Arthroskopie ist auch bei der Behandlung der Kniegelenksluxation hoch, da so insbesondere die Visualisierung und Behandlung intraartikulärer Verletzungen von Knorpel und $\mathrm{Me}$ niskus gewährleistet werden kann. Auch die anatomische Rekonstruktion der zentralen Säule ist für den arthroskopisch geschulten Chirurgen deutlich einfacher möglich.

Eine konsequente regelmäßige Überwachung der Weichteile muss jedoch dabei beachtet werden, da es aufgrund von Verletzung der Kapsel während der Arthroskopie zu einer Erhöhung des intrakompartimentellen Druckes im Unterschenkel kommen kann und die große Gefahr der Entwicklung eines Kompartmentsyndroms besteht.

Da von peripher nach zentral rekonstruiert werden sollte, kann der periphere Zugang genutzt werden, den kontinuierlichen Wasserablauf zu gewährleisten, wodurch das Risiko eines Kompartments im Rahmen der Arthroskopie kontrolliert werden kann. Es ist $\mathrm{zu}$ empfehlen mit einem grundsätzlich niedrigen Wasserdruck zu arbeiten [2]. Eine Blutsperre wird regelhaft angelegt, aber nur sequenziell genutzt.

\section{Operatives Vorgehen}

Der kniegelenkübergreifende Fixateur externe ist im Falle der nicht sicher in Reposition haltbaren Luxationen, bei Kompartmentsyndrom und zur Reposition nach gefäßchirurgischem Eingriff $\mathrm{zu}$ verwenden. Die Anlage sollte dabei stets unter fluoroskopischer Kontrolle erfolgen, um eine Fixation in der hinteren Schublade zu vermeiden. Die Ausbehandlung im Fixateur oder die Anlage eines Bewegungsfixateurs sollte nur in Ausnahmefällen z. B. bei starker Polytraumatisierung erfolgen.

Auch wenn isolierte periphere Kapselbandverletzungen des Kniegelenks ein gewisses Selbstheilungspotenzial haben, ist dies im Rahmen von Luxationsverletzungen eher als gering einzuschätzen. Aus diesem Grund werden primäre, anatomische schichtweise Rekonstruktionen des peripheren Kapselbandapparates mit
Nähten, Fadenankersystemen, Krampen etc. empfohlen. Auch die Verstärkung der Rekonstruktionen mit ultrareißfestem Fadenmaterial, dem sog. LigamentBracing, zeigt vielversprechende Ergebnisse [8].

Abhängig von der anatomischen Rekonstruktion und der hieraus resultierenden Stabilität können primäre Bandersatzplastiken mittels Auto- oder Allograft eine deutliche Zunahme der Stabilität ermöglichen. Sofern eine primäre Rekonstruktion ggf. mit Bandersatz der peripheren und zentralen Strukturen angestrebt wird, muss das Sehnenmanagement bereits präoperativ durchdacht werden. So empfiehlt sich das sterile Abwaschen der Gegenseite, um ggf. vielfältigere Optionen bei der Transplantatwahl zu haben.

Dies spielt insbesondere bei Verletzungen des medialen Kapselbandapparates eine Rolle, da hier eine Zerreißung oder Verletzung der Hamstring-Sehnen bestehen kann und diese daher potenziell nicht als Transplantat zur Verfügung stehen könnten. Auch wird eine zusätzliche Schwächung der sekundären dynamischen Stabilisatoren des medialen Kapselbandapparates durch die Sehnenentnahme der betroffenen Seite ggf. vermindert. Die Rekonstruktion der Kapselbandstrukturen erfordert, mit den entsprechenden Bandersatztechniken vertraut $\mathrm{zu}$ sein.

Dies gilt insbesondere für primäre Versorgungsansätze der Kreuzbänder. Vorteile der primären zentralen Rekonstruktion liegen in einer höheren Stabilität. Die Häufigkeit verbleibender Instabilitäten und Folgeoperation nach zentralen Nähten ist gegenüber primären Bandersatzplastiken deutlich höher [12].

Zusammenfassend sind sowohl die Behandlung der Multiligamentverletzungen als auch die Verwendung verschiedener operativerer Techniken stark variierend und müssen individuell angepasst werden. Primäre Rekonstruktionen und Bandersatzplastiken sollten anatomisch erfolgen und eine frühfunktionelle Nachbehandlung ermöglichen.

Eine weitere Übersicht der evidenzbasierten Therapieempfehlungen bei Kniegelengluxationsverletzungen bietet die Leitlinie der Arbeitsgemeinschaft der 


\section{Infobox 2 Phasen der Rehabili- tation nach komplexer Kniegelenk- rekonstruktion}

- Schutz der Bandrekonstruktion (6 Wochen postoperativ)

- Ziel: Weichteilkonditionierung, Verbesserung der Beweglichkeit (,range of motion" [ROM])

- Funktionsverbesserung (7. bis 12 . Woche postoperativ)

- Ziel: Verbesserung der physiologischen ROM

- Kräftigung

(12. bis 24. Woche postoperativ)

- Ziel: Muskuläre Kräftigung, Verbesserung Funktion und Sensomotorik

- "Return to activity"

(ab 24. Woche postoperativ)

- Ziel: Soziale Reintegration und Rückkehr zur gewohnten Aktivität und zum Sport

Wissenschaftlichen Medizinischen Fachgesellschaften (AWFM) (•Infobox 1).

\section{Nachbehandlung}

Neben der operativen Therapie besitzt die postoperative Rehabilitation einen großen Stellenwert, um ein gutes Therapieergebnis zu erzielen. Die Schwierigkeit in der Nachbehandlung der Kniegelenksluxationen liegt im Verhältnis von Immobilisation zur Unterstützung der Heilung und funktioneller Therapie zur Verbesserung des Bewegungsausmaßes und Verhinderung der Arthrofibrose. Die Nachbehandlung sollte sich an das Therapiekonzept der führenden rekonstruierten Bandstrukturen anpassen meist ist dies das hintere Kreuzband (HKB). Die auf Anspruch und Fähigkeit des Patienten angepasste Rehabilitation gliedert sich grob in die in $\mathbf{0}$ Infobox 2 aufgeführten 4 Phasen [10].

Grundsätzlich sollten die Leitsätze "Stabilität vor Beweglichkeit" und „Beweglichkeit vor Kraft" beachtet werden, um verbleibende Instabilität und Einschränkung der Beweglichkeit zu vermeiden.

\section{„Stabilität vor Beweglichkeit"}

Insgesamt stellt die Nachbehandlung von komplexen Knieverletzungen sowohl für den Patienten als auch für den Therapeuten eine Herausforderung dar. Eine
Aufklärung über Dauer, Wichtigkeit und Intensität sollte daher schon im Rahmen des Klinikaufenthalts erfolgen und betont werden.

\section{Outcome}

Auch bei bestmöglicher operativer Versorgung und Nachbehandlung kann bei Kniegelenksluxationen eine chronische Instabilität verbleiben.

Frosch et al. [6] beschreiben in einer Metaanalyse, dass sowohl nach einals auch zweizeitigem operativem Vorgehen in $20 \%$ der Fälle eine chronische Instabilität verbleibt. Vor allem Patienten mit Kniegelenksluxation nach Hochrasanztraumen haben häufiger ein schlechteres Outcome, da diese Verletzungen vielfach im Rahmen von Mehrfachverletzungen auftreten, bei denen mögliche lebensbedrohliche Traumen bei der Primärversorgung im Vordergrund stehen [13]. Auch eine neurovaskuläre Beteiligung geht mit schlechteren Ergebnissen einher. Insgesamt erreichen nur $50 \%$ der Patienten eine nahezu normale Alltagsaktivität, wobei insgesamt $43 \%$ der Patienten 10 Jahre nach Kniegelenksluxation eine Arthrose im betroffenen Knie entwickeln $[4,14]$.

\section{Fazit für die Praxis}

- Bei Verletzung von 2 der 4 Hauptsäulen der Kniegelenkstabilität und der Kniegelenkkapsel muss von einer Luxation bzw. Subluxation des Kniegelenks ausgegangen werden.

- Bei CBI <0,9 oder klinischem Verdacht eines Gefäßverschlusses sollte eine CT-Angiographie durchgeführt werden.

- Nach gefäßchirurgischem Eingriff muss eine sichere Reposition im Fixateur externe oder durch Rekonstruktion gewährleistet werden.

- Die operative Versorgung ist bei KD II-V-Verletzungen der konservativen Therapie vorzuziehen.

- Die aktive Dorsiflexion des Fußes, der Großzehen sowie das sensible Gebiet des N. peroneus am Fußrücken müssen untersucht und dokumentiert werden.
- Trotz guter Therapie sind frühzeitige Komplikationen, Auftreten von Arthrose und persistierende Instabilität möglich. Eine Aufklärung des Patienten über die Schwere der Verletzung und drohende Folgen sollte frühzeitig erfolgen.

\section{Korrespondenzadresse

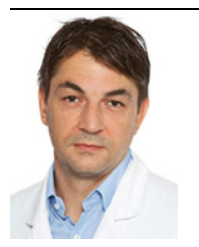 \\ PD Dr. S. T. Shafizadeh \\ Klinik für Orthopädie, Unfallchirurgie und Sporttraumatologie, Lehrstuhl der Universität Witten-Herdecke, Klinken der Stadt Köln - Klinikum Merheim Köln Ostmerheimerstr. 200 51109 Köln, Deutschland Shafizadehs@ kliniken-koeln.de}

\section{Einhaltung ethischer Richtlinien}

Interessenkonflikt. S.T. Shafizadeh, B. Bouillon, J.H. Naendrup, V. Jaecker und T.R. Pfeiffer geben an, dass kein Interessenkonflikt besteht.

Dieser Beitrag beinhaltet keine von den Autoren durchgeführten Studien an Menschen oder Tieren.

The supplement containing this article is not sponsored by industry.

\section{Literatur}

1. Azar FM, Brandt JC, Miller RH (2011) Ultra-lowvelocity knee dislocations. Am J Sports Med 39(10):2170-2174

2. Boyce RH, Singh K, Obremskey WT (2015) Acute management of traumatic knee dislocations for the generalist. J Am Acad Orthop Surg 23(12):761-768. https://doi.org/10.5435/JAAOSD-14-00349

3. Burrus MT, Werner BC, Griffin JW (2016) Diagnostic and management strategies for Multiligament knee injuries: a critical analysis review. JBJS Rev 4(2):1. https://doi.org/10.2106/JBJS.RVW.0.00020

4. Engebretsen L, Risberg MA, Robertson B (2009) Outcome after knee dislocations: a 2-9 years follow-up of 85 consecutive patients. Knee Surg Sports Traumatol Arthrosc 17(9):1013-1026

5. Fanelli GC, Giannotti BF, Edson CJ (1996) Arthroscopically assisted combined posterior cruciate ligament/posterior lateral complex reconstruction. Arthroscopy 12(5):521-530. https://doi.org/ 10.1016/50749-8063(96)90189-9

6. Frosch K-H, Preiss A, Heider S (2013) Primary ligament sutures as a treatment option of knee dislocations: a meta-analysis. Knee Surg Sports Traumatol Arthrosc 21(7):1502-1509

7. Harner CD, Waltrip RL, Bennett CH (2004) Surgical management of knee dislocations. J Bone Joint Surg Am 86-A(2):262-273 
8. Heitmann M, Gerau M, Hötzel J (2014) Ligament bracing - Augmentierte Primärnaht bei multiligamentären Verletzungen des Kniegelenks. Oper Orthop Traumatol 26(1):19-29

9. Kennedey JC (1963) Complete dislocation of the knee joint. JBone Joint Surg Am 45(5):889-904

10. Klose M, Diermeier T, Beitzel K (2017) Rehabilitation nach multiligamentären Rekonstruktionen. Arthroskopie 30(1):60-66

11. Levy BA, Dajani KA, Whelan DB (2009) Decision making in the multiligament-injured knee: an evidence-based systematic review. Arthroscopy 25(4):430-438. https://doi.org/10.1016/j.arthro. 2009.01.008

12. Levy BA, Fanelli GC, Whelan DB (2009) Controversies in the treatment of knee dislocations and multiligament reconstruction. J Am Acad Orthop Surg 17(4):197-206

13. Levy NM, Krych AJ, Hevesi M (2015) Does age predict outcome after multiligament knee reconstruction for the dislocated knee? 2- to 22-year follow-up. Knee Surg Sports Traumatol Arthrosc 23(10):3003-3007

14. Moatshe G, Dornan GJ, Ludvigsen T (2017) High prevalence of knee osteoarthritis at a minimum 10-year follow-up after knee dislocation surgery. Knee Surg Sports Traumatol Arthrosc. https://doi. org/10.1007/s00167-017-4443-8

15. Molund M, Engebretsen L, Hvaal K (2014) Posterior tibial tendon transfer improves function for foot drop after knee dislocation. Clin Orthop Relat Res 472(9):2637-2643

16. Mook WR, Ligh CA, Moorman CT (2013) Nerve injury complicating multiligament knee injury: current concepts and treatment algorithm. J Am Acad Orthop Surg 21(6):343-354. https://doi.org/ 10.5435/JAAOS-21-06-343

17. Mook WR, Miller MD, Diduch DR (2009) Multipleligament knee injuries: a systematic review of the timing of operative intervention and postoperative rehabilitation. JBone Joint Surg Am 91(12):2946-2957. https://doi.org/10.2106/JBJS. H.01328

18. Natsuhara KM, Yeranosian MG, Cohen JR (2014) What is the frequency of vascular injury after knee dislocation? Clin Orthop Relat Res 472(9):2615-2620. https://doi.org/10.1007/ s11999-014-3566-1

19. Nicandri GT, Slaney SL, Neradilek MB (2011) Can magnetic resonance imaging predict posterior drawer laxity at the time of surgery in patients with knee dislocation or multiple-ligament knee injury? Am J Sports Med 39(5):1053-1058. https://doi. org/10.1177/0363546510391154

20. Patterson BM, Agel J, Swiontkowski MF et al (2007) Knee dislocations with vascular injury: outcomes in the lower extremity assessment project (LEAP) study. JTrauma 63(4):855-858

21. Peskun CJ, Whelan DB (2011) Outcomes of operative and nonoperative treatment of multiligament knee injuries: an evidence-based review. Sports Med Arthrosc 19(2):167-173. https://doi.org/10. 1097/JSA.0b013e3182107d5f

22. Rihn JA, Groff YJ, Harner CD et al (2004) The acutely dislocated knee: evaluation and management. J Am Acad Orthop Surg 12(5):334-346

23. Schenck R (2003) Classification of knee dislocations. Oper Tech Sports Med 11(3):193-198. https:// doi.org/10.1053/otsm.2003.35918

24. Seroyer ST, Musahl V, Harner CD (2008) Management of the acute knee dislocation: the Pittsburgh experience. Injury 39(3):710-718. https://doi.org/ 10.1016/j.injury.2007.11.022
25. Shelbourne KD, Klootwyk TE (2000) Low-velocity knee dislocation with sports injuries. Treatment principles. Clin Sports Med 19(3):443-456. https:// doi.org/10.1016/S0278-5919(05)70217-9

26. Sillanpaa PJ, Kannus P, Niemi ST et al (2014) Incidence of knee dislocation and concomitant vascular injury requiring surgery: a nationwide study. J Trauma Acute Care Surg 76(3):715-719. https://doi.org/10.1097/TA.0000000000000136 